\title{
Parkin, PINK1, and DJ-1 form a ubiquitin E3 ligase complex promoting unfolded protein degradation
}

\author{
Hui Xiong, ${ }^{1}$ Danling Wang, ${ }^{1,2}$ Linan Chen, ${ }^{3}$ Yeun Su Choo, ${ }^{1}$ Hong Ma, ${ }^{1}$ Chengyuan Tang, ${ }^{1,2}$ \\ Kun Xia, ${ }^{2}$ Wei Jiang, ${ }^{1}$ Ze'ev Ronai, ${ }^{1}$ Xiaoxi Zhuang, ${ }^{3}$ and Zhuohua Zhang ${ }^{1,2}$ \\ ${ }^{1}$ Burnham Institute for Medical Research, La Jolla, California, USA. ${ }^{2}$ State Key Laboratory of Medical Genetics, Central South University, \\ Changsha, Hunan, People's Republic of China. ${ }^{3}$ Department of Molecular Pharmacology, University of Chicago School of Medicine, Chicago, Illinois, USA.
}

\begin{abstract}
Mutations in PARKIN, pten-induced putative kinase 1 (PINK1), and DJ-1 are individually linked to autosomal recessive early-onset familial forms of Parkinson disease (PD). Although mutations in these genes lead to the same disease state, the functional relationships between them and how their respective disease-associated mutations cause PD are largely unknown. Here, we show that Parkin, PINK1, and DJ-1 formed a complex (termed PPD complex) to promote ubiquitination and degradation of Parkin substrates, including Parkin itself and Synphilin-1 in neuroblastoma cells and human brain lysates. Genetic ablation of either Pink1 or $D j-1$ resulted in reduced ubiquitination of endogenous Parkin as well as decreased degradation and increased accumulation of aberrantly expressed Parkin substrates. Expression of PINK1 enhanced Parkin-mediated degradation of heat shock-induced misfolded protein. In contrast, PD-pathogenic Parkin and PINK1 mutations showed reduced ability to promote degradation of Parkin substrates. This study identified a functional ubiquitin E3 ligase complex consisting of PD-associated Parkin, PINK1, and DJ-1 to promote degradation of un-/misfolded proteins and suggests that their PD-pathogenic mutations impair E3 ligase activity of the complex, which may constitute a mechanism underlying PD pathogenesis.
\end{abstract}

\section{Introduction}

Parkinson disease (PD) is a progressive neurodegenerative movement disorder affecting approximately $2 \%$ of the population over 65 years of age. It is pathologically characterized by pronounced loss of dopaminergic neurons in the substantia nigra of the midbrain and formation of ubiquitin-positive Lewy body aggregates $(1,2)$. Although administration of L-DOPA temporarily relieves parkinsonism, no treatment is currently available to prevent disease progression and neurodegeneration. Understanding the molecular basis of PD is likely to facilitate development of effective therapies of the disease.

Most PD cases are sporadic. Nevertheless, mutations in at least 7 genes are linked to early onset familial form PD, including mutations in a-synuclein, ubiquitin carboxyl-terminal esterase L1 (uchL1), and leucine-rich repeat kinase 2 (LRRK2) for autosomal dominant cases and mutations in Parkin, pten-induced putative kinase 1 (PINK1), DJ-1, and ATPase type 13A2 (ATP13A2) for autosomal recessive cases (1-6). Characterization of these genes has provided important insights into PD pathogenesis. For example, $\alpha$-synuclein is a major structural component of Lewy bodies in PD (7). PD-associated $\alpha$-synuclein mutant proteins show increased propensity to self-aggregate to form oligomeric species and Lewy body-like fibrils compared with WT $\alpha$-synuclein, directly linking disease-associated $\alpha$-synuclein mutant proteins to PD pathology $(8,9)$. However, the functional relationships of these genes and how their respective disease-associated mutations cause selective

Authorship note: Hui Xiong and Danling Wang contributed equally to this work. Conflict of interest: The authors have declared that no conflict of interest exists.

Nonstandard abbreviations used: GST, glutathione-S-transferase; PD, Parkinson disease; PINK1, pten-induced putative kinase 1; PPD, Parkin/PINK1/DJ-1 (complex); UPS, ubiquitin-proteasome system; VSVG, vesicular stomatitis virus glycoprotein. Citation for this article: J. Clin. Invest. 119:650-660 (2009). doi:10.1172/JCI37617. neuronal loss and Lewy body formation remains largely unknown, even though mutations of these genes cause the same disease.

Among the genes linked to the autosomal recessive early onset familial form of PD, Parkin encodes a protein with E3 ligase activity, mediating ubiquitination and degradation of multiple proteins (5, 10-12). Sequence analysis strongly suggests that PINK1 is a putative kinase with 2 identified substrates (13-15). DJ-1 protects cells from oxidative stress both in transfected cells and in Drosophila (16-19). Inactivation of Parkin or Dj-1 in mouse and PINK1 in Drosophila results in mitochondrial dysfunction and increased sensitivity to oxidative stress (20-25). Together, Parkin, PINK1, and DJ-1 likely protect against oxidative stress in cells via a common mechanism, suggesting potential functional interactions among the 3 proteins.

In this study, we aimed to investigate the functional relationship of Parkin, PINK1, and DJ-1. Our results demonstrate that Parkin, PINK1, and DJ-1 form what we believe is a novel E3 complex that promotes ubiquitination and degradation of aberrantly expressed and heat shock-induced Parkin substrates, Parkin and Synphilin-1. Pathogenic PINK1 or Parkin mutants showed impaired ability to degrade Parkin and Synphilin-1. These results suggest that the Parkin/PINK1/DJ-1 (PPD) complex plays an important role in degradation of un-/misfolded Parkin substrates.

\section{Results}

Complex formation of Parkin, PINK1, and DJ-1 in vitro and in vivo. To examine whether proteins encoded by PD-associated Parkin, PINK1, or $D J-1$ regulate a common functional pathway via complex formation, we coexpressed vesicular stomatitis virus glycoprotein-tagged (VSVG-tagged) Parkin (ParkinWT), flag-tagged PINK1 (PINK1WT), and myc-tagged DJ-1 (DJ-1WT) in various combinations in SH-SY5Y neuroblastoma cells (Figure 1) and HEK293 cells (data not shown). Immunoprecipitation of cell lysates using corresponding anti-tag 
antibodies revealed that Parkin, PINK1, and DJ-1 were specifically coprecipitated in any combination of 2 or all 3 proteins (Figure 1 , A-F). We next performed in vitro interaction assays using purified flag-myc dual-tagged Parkin, flag-VSVG dual-tagged PINK1, and glutathione-S-transferase-VSVG (GST-VSVG) dual-tagged DJ-1 recombinant proteins (Supplemental Figure 1; supplemental material available online with this article; doi:10.1172/JCI37617DS1). Consistent with the coimmunoprecipitation results, Parkin bound to PINK1, DJ-1, or both (Figure 1G). Likewise, DJ-1 interacted with Parkin, PINK1, or both (Figure 1H). In contrast, none of the 3 proteins bound to control fusion protein KChIP1 (data not shown). These results suggest that Parkin, PINK1, and DJ-1 physically associate. Gel filtration analysis of the complex assembled in vitro suggested that Parkin, PINK1, and DJ-1 form an approximately $200-\mathrm{kDa}$ complex (Supplemental Figure 2). Deletion studies indicated that Parkin, PINK1, and DJ-1 proteins interact with each other via distinct domains (Supplemental Figure 3), further supporting the idea that a stable complex of the 3 proteins forms.

We next examined interaction of endogenous Parkin, PINK1, and DJ-1 in human brain lysates. Immunoprecipitation of endogenous Parkin with an anti-Parkin monoclonal antibody, but not an unrelated control mouse IgG, coprecipitated endogenous PINK1 and DJ-1 (Figure 1I), suggesting that Parkin, PINK1, and DJ-1 form a complex in vivo. We were not able to perform immunoprecipitation of endogenous PINK1 because of unavailability of anti-PINK1 antibodies for immunoprecipitation. To provide further evidence for complex formation in vivo, we immunoprecipitated PINK1 from SH-SY5Y cells stably expressing a low level of flag-tagged PINK1, then determined coprecipitated proteins using mass spectrometry. Three functional classes of proteins were found to interact with PINK1 in cells expressing PINK1 but not in cells transfected with vector alone. These included heat shock protein chaperones, mitochondrial proteins, and proteins involved in the ubiquitin-proteasome pathway (data not shown). In addition, endogenous Parkin and DJ-1 were identified (Supplemental Table 1). Together, Parkin, PINK1, and DJ-1 formed a complex in vitro and in vivo that we henceforth refer to as the PPD complex (Figure 1J).

Intracellular localization of the PPD complex. PINK1 is suggested to be a mitochondrial protein and to localize to mitochondrion. We next determined localization of the PPD complex by immunostaining and cellular fractionation. Immunostaining of SH-SY5Y neuroblastoma cells (data not shown) and cultured primary human neurons showed colocalization of Parkin, PINK1, and DJ-1 largely in the cytoplasm (Supplemental Figure 4). Fractionation analysis of SH-SY5Y cells stably expressing Parkin, PINK1, and DJ-1 detected all 3 proteins in both the mitochondrial and the cytosolic fractions (Figure 2A). Consistent with the immunostaining results, all 3 proteins were more abundant in the cytosolic fraction than in the mitochondrial fraction. Immunoprecipitation of fractionated cell lysates revealed that the majority of PPD complex was present in the cytosolic fraction, while only a minor amount of the PPD complex was detected in the mitochondrial fraction (Figure 2B). Interestingly, the full-length PINK1 (64-kDa fragment) was detected mostly in the mitochondrial fraction, while the proteolytic processed PINK1 fragments $(55 \mathrm{kDa}$ and $48 \mathrm{kDa}$ ) were mainly found in the cytosolic fraction (Figure 2A and Supplemental Figure 5). Furthermore, Parkin coprecipitated with only the 55-kDa PINK1 fragment. Detection of the processed PINK1 mainly in the cytosol indicated that the cytosolic PINK1 was not likely the result of increased expression of exogenous protein. These results suggest that the $55-\mathrm{kDa}$
PINK1 proteolytic fragment was likely the active PINK1 in the PPD complex (Figure 2B). Thus, the PPD complex is mainly localized in cytosolic fraction of the cell.

PPD promotes degradation of Parkin and Synphilin-1 via the ubiquitinproteasome system. Parkin functions as an E3 ubiquitin ligase in the ubiquitin-proteasome system (UPS) (10-12). We next examined whether PINK1 regulates degradation of the previously defined Parkin substrates, Parkin and Synphilin-1 $(11,26)$. Expression of PINK1 in SH-SY5Y (Figure 3, A and B) and HEK293 cells (data not shown) remarkably reduced the steady-state level of Parkin or Synphilin-1 compared with control transfectants. PINK1-induced reduction in Parkin and Synphilin-1 levels was largely rescued by treatment with the proteasome inhibitors MG132 and lactocystin (data not shown) but only slightly inhibited by treatment with the protease inhibitor leupeptin (Figure 3, A and B). Pulse chase analysis showed that Parkin stability was remarkably reduced with PINK1 expression. In the presence of MG132 (5 $\mu \mathrm{M})$, PINK1-promoted Parkin degradation was inhibited, resulting in accumulation of both monomeric and ubiquitinated forms of Parkin (Figure 3, C and D). Thus, PINK1 promotes Parkin and Synphilin-1 degradation primarily via the UPS.

We next investigated ubiquitination of Parkin and Synphilin-1 in the presence of PINK1. Parkin or Synphilin-1 was coexpressed with either PINK1WT or a pathogenic PINK1G309D mutant in SH-SY5Y cells. As anticipated, Parkin levels were remarkably reduced, while ubiquitination of Parkin was substantially increased in cells expressing PINK1WT compared with control transfectants (Figure 4, A and B). Increased Parkin ubiquitination was deemed notable because the level of Parkin in cells expressing PINK1WT was particularly low compared with that seen in cells expressing Parkin alone. PINK1-dependent ubiquitination of Parkin was further supported by reduced detection of monomeric ubiquitin (Figure 4D). Likewise, remarkably reduced levels of Synphilin-1 protein (Figure $4 \mathrm{H}$ ) and monomeric ubiquitin (Figure $4 \mathrm{~J}$ ) were seen in cells expressing Synphilin-1 and PINK1 compared with cells expressing Synphilin-1 alone. Nevertheless, in cells expressing PINK1WT, Synphilin-1 ubiquitination appeared to be less extensive than that seen in control cells, likely due to marked degradation of Synphilin-1 and the fact that little protein was available for immunoprecipitation. These results suggest that PINK1 promotes degradation of Parkin substrates primarily by promoting ubiquitination of Parkin substrates. PINK1 promotes Synphilin-1 degradation in the absence of overexpressed Parkin (Figure 4, G and $\mathrm{H}$ ), most likely through the activity of endogenous Parkin in SH-SY5Y cells. Coexpression of Parkin increased ubiquitination and the steady-state level of PINK1 (Figure 4, E, F, K, and L). Thus, PINK1 is unlikely a Parkin substrate for UPS degradation.

Roles of PINK1 in promoting Parkin ubiquitination were further verified by an in vitro Parkin auto-ubiquitination assay using affinity-purified Parkin and PINK1 fusion proteins. In the presence of E1 or E2 alone, little ubiquitinated Parkin was detected (Figure 5). Consistent with a previous report that auto-ubiquitinated Parkin is limited in vitro (12), ubiquitination of Parkin was not substantially increased when both E1 and E2 were included in the assay. In contrast, Parkin ubiquitination was remarkably enhanced by adding PINK1WT. Increased Parkin ubiquitination was likely specifically induced by PINK1 because the pathogenic PINK1G309D protein had a much smaller effect than PINK1WT. PINK1WT promoted ubiquitination of a pathogenic Parkin mutant (R42P) with notably lower potency than it did for ParkinWT (Figure 5). Consis- 

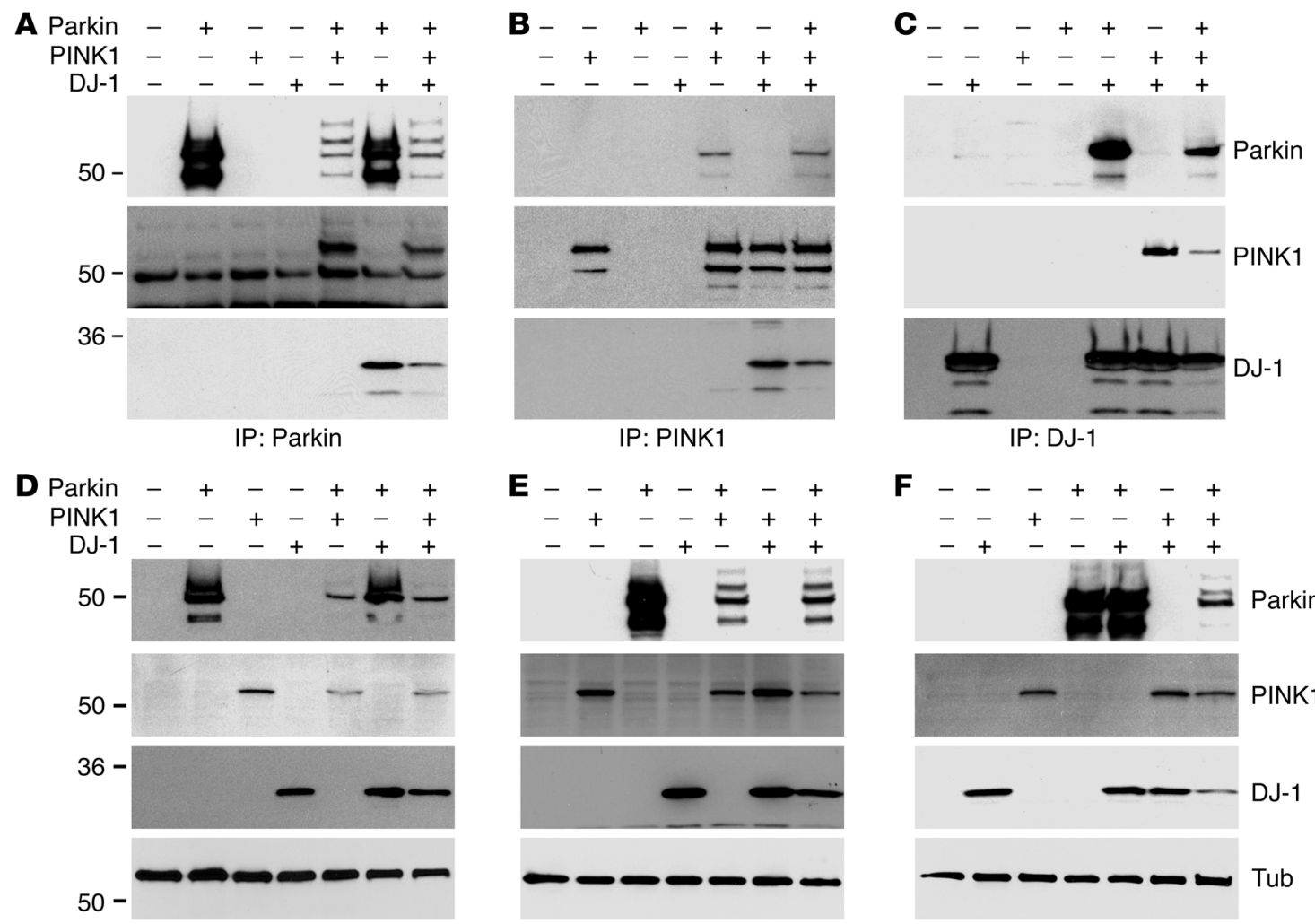

$$
\text { IP: PINK1 }
$$

$$
\text { IP: DJ-1 }
$$
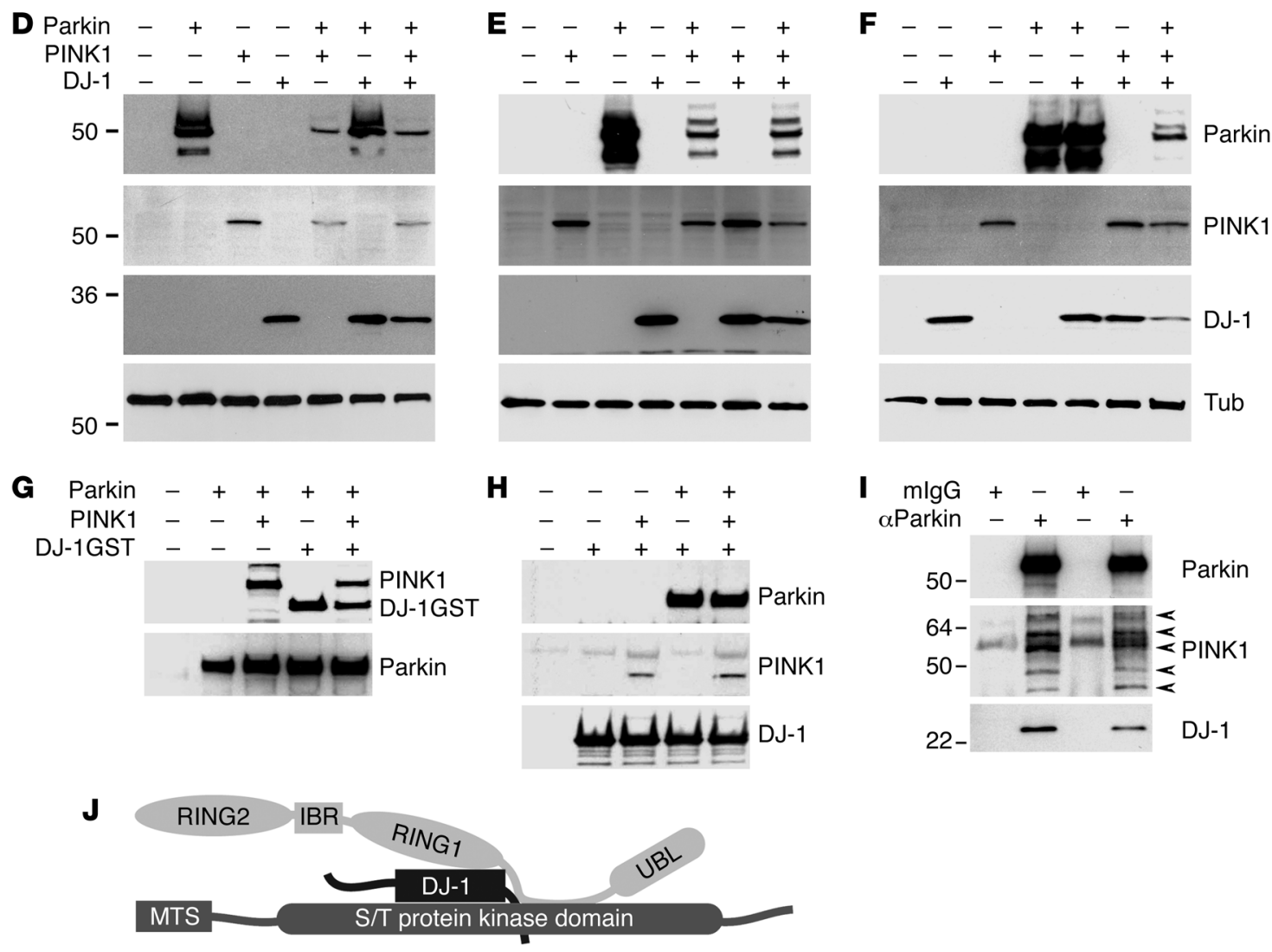

Figure 1

Complex formation of Parkin, PINK1, and DJ-1. (A-F) Association of Parkin, PINK1, and DJ-1 in transfected cells. Parkin-VSVG, PINK1-flag, and DJ-1-myc were expressed in various combinations and immunoprecipitated with antibodies to the corresponding tag, followed by detection of coprecipitation of PINK1 and DJ-1 (A), Parkin and DJ-1 (B), and Parkin and PINK1 (C), respectively. (D-F) Inputs of Parkin (D), PINK1 (E), and DJ-1 (F). Note that cotransfection of PINK1 significantly reduced Parkin levels in lysates. Tub, cytosolic marker tubulin. (G and $\mathbf{H})$ In vitro assembly of the PPD complex. Affinity-purified Parkin-myc-flag (Parkin), PINK1-VSVG-flag (PINK1), and GST-DJ-1-VSVG (DJ-1GST) were incubated in various combinations, followed by precipitation with either anti-myc agarose $(\mathbf{G})$ or GST agarose $(\mathbf{H})$. Precipitates were detected with an anti-VSVG antibody to detect both PINK1 and DJ-1-GST (G), an anti-Parkin antibody to detect Parkin (H), an anti-PINK1 antibody to detect PINK1 $(\mathbf{H})$, or an anti-DJ-1 antibody to detect DJ-1 (H). (I) Association of Parkin, PINK1, and DJ-1 in vivo. Lysates of human brain cortex from 2 unrelated individuals (lanes 1 and 2 for one individual, lanes 3 and 4 for the other) were immunoprecipitated with an anti-Parkin monoclonal antibody ( $\alpha$ Parkin) or control mouse IgG (mlgG), followed by immunoblotting with a polyclonal anti-Parkin antibody, a polyclonal anti-PINK1 antibody, or a monoclonal anti-DJ-1 antibody. Multiple endogenous PINK1 proteolytic bands were detected (arrows). (J) A schematic illustration of interaction among PPD complex components. IBR, in between RING fingers; MTS, mitochondrial targeting sequence; UBL, ubiquitin-like.

tent with this observation, PINK1G309D did not promote ubiquitination and degradation of Parkin and Synphilin-1 in transfected cells (Figure 4, B and H). The results suggest that PD-pathogenic PINK1 and Parkin mutants impair PPD complex activity.
To determine the potential role of DJ-1 in the PPD complex, WT DJ-1 (DJ-1WT) or a pathogenic loss-of-function mutant DJ-1L166P was included in some of the experiments shown in Figure 4. Parkin ubiquitination was consistently slightly increased in the presence of 


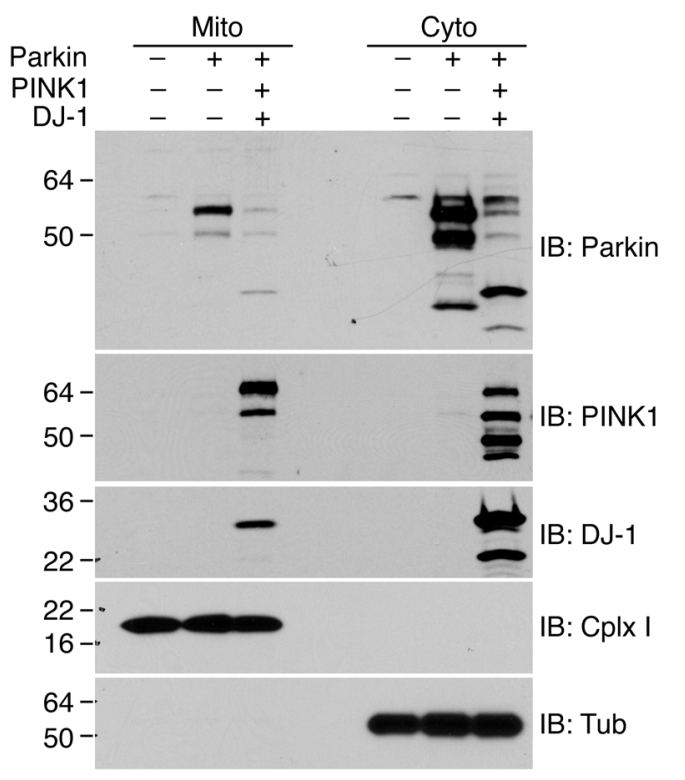

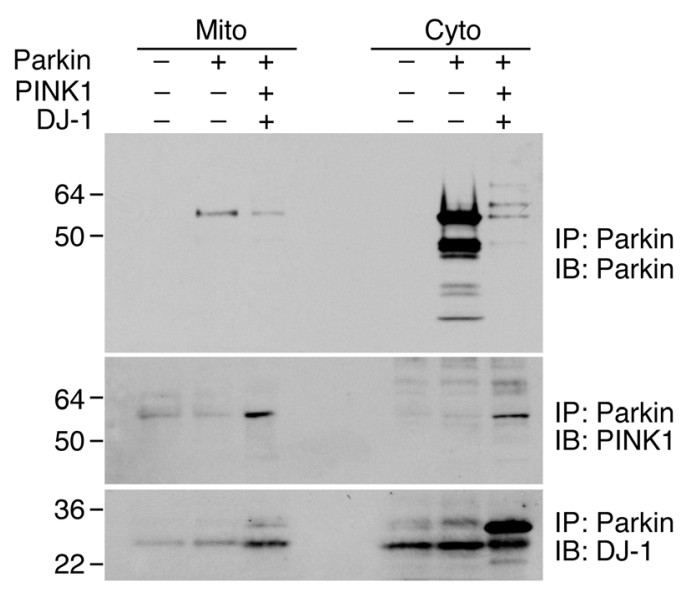

Figure 2

Detection of the PPD complex in both mitochondria and cytosolic fractions. Cells expressing Parkin alone or a combination of Parkin, PINK1, and DJ-1 were fractionated to mitochondrial (Mito) and cytosolic (Cyto) fractions. Left: Expression of Parkin, PINK1, DJ-1, mitochondria marker complex I (Cplx I), and cytosolic marker tubulin. Right: Coimmunoprecipitation of Parkin with PINK1 and DJ-1 in mitochondria and cytosolic fractions.
DJ-1WT despite the fact that steady-state levels of Parkin were not obviously altered (Figure 4A). Similar results were obtained with Synphilin-1 (data not shown). In agreement with our recent finding that PINK1 levels are stabilized by DJ-1WT but not by PD-associated DJ-1A39S (27), the steady-state level of PINK1 was consistently increased in cells expressing DJ-1WT (Figure 4F). Therefore, DJ-1 likely modulates PINK1 in the PPD complex.

Accumulation of aberrantly expressed Parkin in PINK1- or DJ-1-deficient cells. Parkin can act as a substrate of itself. We examined roles of endogenous PINK1 and DJ-1 in promoting degradation and ubiquitination of Parkin using PINK1- or DJ-1-deficient mouse brains and cells generated from mouse embryos in which PINK1 or DJ-1 was genetically deleted (28). Ablation of either PINK1 or DJ-1 had little effect on steady-state level of endogenous Parkin (data not shown). We next determined ubiquitination of endogenous Parkin using mouse brain slice cultures that were generated from either WT or PINK1-null mice. Endogenous Parkin ubiquitination was detected by immunoprecipitation of Parkin followed by immunoblotting detection of ubiquitin. In control WT mouse brain slices, polyubiquitination of Parkin was detected as high molecular weight-smeared protein species (Figure 6). In contrast, polyubiquitinated Parkin in PINK1-null brain slices was remarkably reduced. Heat shock treatment enhanced Parkin ubiquitination in WT mouse brain slices but not in PINK1-null mouse brain slices. We did not observe increased accumulation of endogenous Parkin in PINK1-null brain slice cultures. One possible explanation is that an alternative pathway is involved in degradation of non-ubiquitinated Parkin. Moreover, treatment of HEK293 cells expressing Synphilin-1 with a mixture of Parkin-specific siRNA oligos inhibited Parkin expression, rescued PINK1-induced Synphilin-1 degradation, and resulted in accumulation of Synphilin-1 (Supplemental Figure 6, A and C). Thus, PINK1 promotes Synphilin-1 degradation via a Parkin-mediated mechanism. These results suggest that endogenous PINK1 plays an important role in Parkin E3 ligase-mediated protein ubiquitination under both normal and stress conditions.

Since heat shock stress induces protein un-/misfolding and Parkin-mediated protein ubiquitination (Figure 6), we further examined the hypothesis that the PPD complex mediates ubiquitina- tion and degradation of un-/misfolded proteins. Overexpression of human Parkin in PINK1-null cells resulted in very significant accumulation of Parkin compared with that seen in control WT cells (Figure 7B). Likewise, increased Parkin detection was seen in DJ-1-null cells overexpressing Parkin compared with matched control cells (Figure 7C). Nevertheless, level of Parkin in DJ-1-null cells was consistently lower than that seen in PINK1-null cells. Pulse chase analysis revealed that Parkin was more stable in both PINK1-null cells (half-life of $4 \mathrm{~h}$ ) and DJ-1-null cells (half life of $2-3 \mathrm{~h}$ ) than in respective WT controls (half-life of $<1 \mathrm{~h}$ ) (Figure 7 , $\mathrm{C}-\mathrm{G})$. These results suggest that the PPD complex is involved in degradation of aberrantly expressed un-/misfolded proteins.

Together, these results suggest that Parkin, PINK1, and DJ-1 are essential components of the PPD E3 ligase activity and that the complex likely plays an important role in degradation of un-/misfolded Parkin substrates.

The PPD complex promotes degradation of un-/misfolded Parkin induced by heat shock stress. To further determine the role of the PPD complex in promoting degradation of un-/misfolded proteins, we examined Parkin degradation after heat shock stress. In SH-SY5Y cells expressing Parkin alone, heat shock stress resulted in increased ubiquitination and accumulation of Parkin, likely un-/misfolded Parkin (Figure 8, A and B). The increased detection of Parkin ubiquitination was likely due to increased Parkin accumulation. In contrast, PINK1 expression not only abolished the Parkin accumulation induced by heat shock but also further promoted Parkin degradation (Figure 8B). These findings are consistent with increased endogenous Parkin ubiquitination by heat shock treatment (Figure 6) and provide further evidence that the PPD complex functions to promote degradation of un-/misfolded proteins.

PD-pathogenic Parkin and PINK1 mutants impair substrate degradation. We next examined the effect of PD-pathogenic mutants of PINK1 and Parkin on the function of the PPD complex. PINK1G309D and Parkin $\Delta$ E4 were able to form a complex with ParkinWT and PINK1WT, respectively (Figure 9, A and B). Compared with PINK1WT-promoted degradation of ParkinWT, PINK1WT poorly promoted degradation of pathogenic Parkin- 


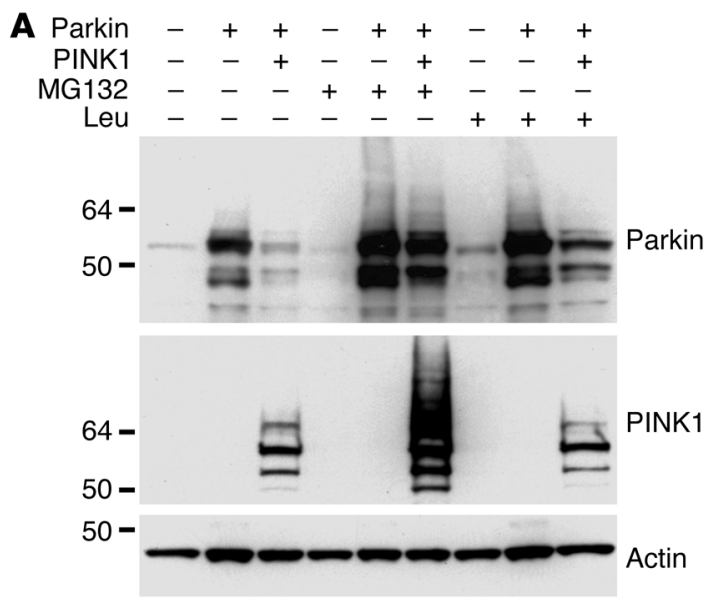

C Chase time (h) $\quad 0 \quad 0.5 \quad 1 \quad 2 \quad 4 \quad 8$

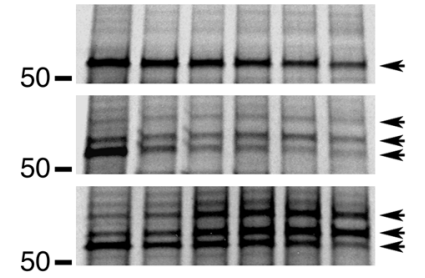

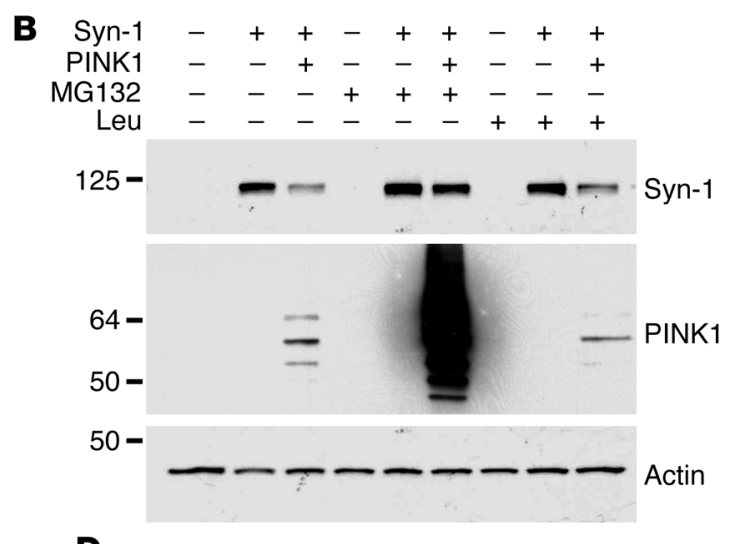

D

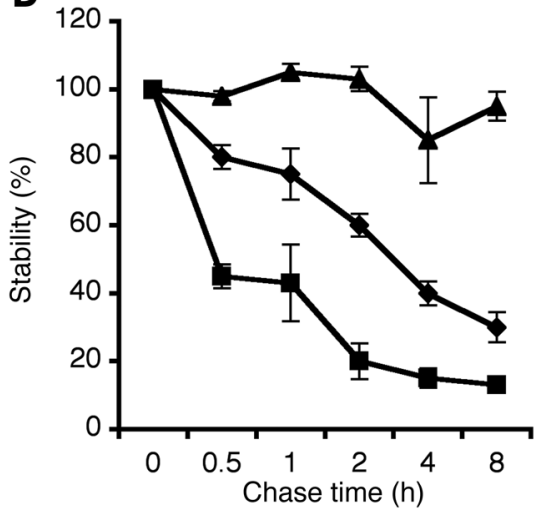

Figure 3

PINK1 promotes proteasomal degradation of Parkin and Synphilin-1. (A and B) SH-SY5Y cells coexpressing PINK1-flag and Parkin-VSVG (A) or PINK1-flag and Synphilin-1-EGFP (Syn-1; B) were treated with either MG132 or leupeptin (Leu). Steady-state levels of Parkin, Synphilin-1, PINK1, and actin (loading control) are shown. (C and D) Expression of PINK1 reduced Parkin stability via the proteasomal pathway. Cells transfected with Parkin alone (C; top panel), Parkin and PINK1 (C; middle panel), or Parkin and PINK1 with MG132 treatment (C; bottom panel) were pulse-labeled, followed by chasing for the indicated time intervals. Levels of Parkin were detected by immunoprecipitation. Results from a representative experiment (C) and quantitation of 3 independent experiments are shown (D). Multiple Parkin bands likely representing ubiquitinated Parkin were detected in the presence of PINK1 (arrows). (D) Diamonds indicate Parkin alone, squares indicate Parkin and PINK1, and triangles indicate Parkin and PINK1 with MG132 treatment.

R42P, -T240W, and - $\Delta \mathrm{E} 4$ (Figure 9, C and E; $P<0.001$ ). Likewise, pathogenic PINK1G309D, PINK1T313M, and PINK1P399L had less of an effect on the steady-state levels (Figure 9, D and F; $P<0.001$ ) or on ubiquitination (data not shown) of ParkinWT and Synphilin-1 compared with PINK1WT. Consistent with a previous study, expression of some pathogenic Parkin and PINK1 mutants was low compared with their WT counterparts in transfected cells (29). Nevertheless, low levels of PD-pathogenic PINK1 mutant proteins unlikely accounted for their reduced ability to promote degradation of Parkin substrates. In in vitro ubiquitination assays, amounts of PINK1G309D mutant protein equivalent to those of WT protein failed to promote comparable Parkin ubiquitination (Figure 5B). Thus, pathogenic Parkin and PINK1 mutant proteins impair substrate degradation likely through reduced PPD complex activity.

\section{Discussion}

We have identified in this study what we believe is a novel functional E3 ligase complex designated as the PPD complex, consisting of 3 PD-associated proteins, Parkin, PINK1, and DJ-1. We further demonstrated that the PPD E3 ligase complex plays an important role in promoting degradation of un-/misfolded Parkin substrates.
PD-pathogenic mutants of Parkin and PINK1 impaired E3 ligase activity of the PPD complex. Our results suggest multiple PD-linked genes function in PD pathogenesis via a common mechanism.

The significance of this study is the finding that 3 PD-related proteins physically interact and form a functional E3 ligase complex to regulate UPS-mediated protein degradation. The findings suggest a function of PINK1 and DJ-1 that is independent of maintaining mitochondrial homeostasis. In our investigation we used 4 different methods to determine complex formation by Parkin, PINK1, and DJ-1 in vitro and in vivo. These include coimmunoprecipitation in cells overexpressing the 3 proteins, in vitro complex assembly using purified recombinant proteins, coimmunoprecipitation in human brain lysates, and immunoprecipitation followed by mass spectrometry analysis. Our findings provide biochemical and mechanistic evidence to support previous reports of genetic interaction of Parkin and PINK1 in Drosophila $(22,23,25)$ and association of digenic mutations of PINK1 and DJ-1 with early onset familial PD cases (27). Nevertheless, the PPD complex is not necessarily the only important one involving PINK1, given that mass spectrometry analysis identifies multiple PINK1 interacting proteins. The E3 ligase activity of the PPD complex was further shown in trans- 


$\begin{array}{rccccccccccc}\text { Parkin } & - & - & + & + & + & + & + & + & + & + & + \\ \text { PINK1 } & - & - & - & + & - & + & + & - & - & - & - \\ \text { PINK1m } & - & - & - & - & + & - & - & + & + & - & - \\ \text { DJ-1 } & - & - & - & - & - & + & - & + & - & + & - \\ \text { DJ-1m } & - & - & - & - & - & - & + & - & + & - & + \\ \text { Ub } & - & + & + & + & + & + & + & + & + & + & +\end{array}$

A
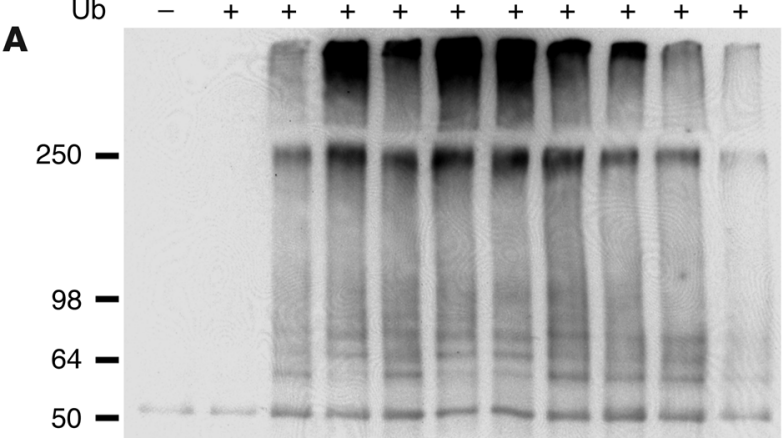

B
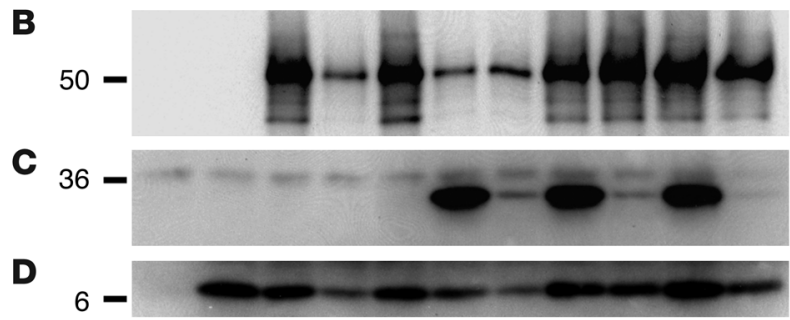
IB. DJ-1

E

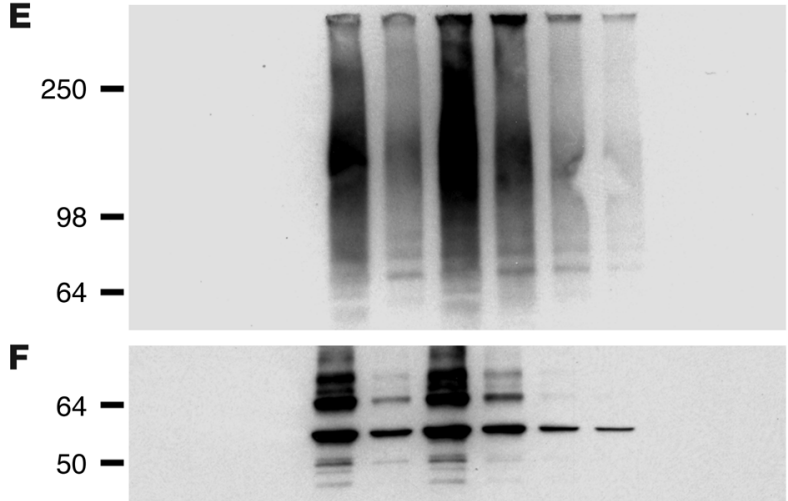

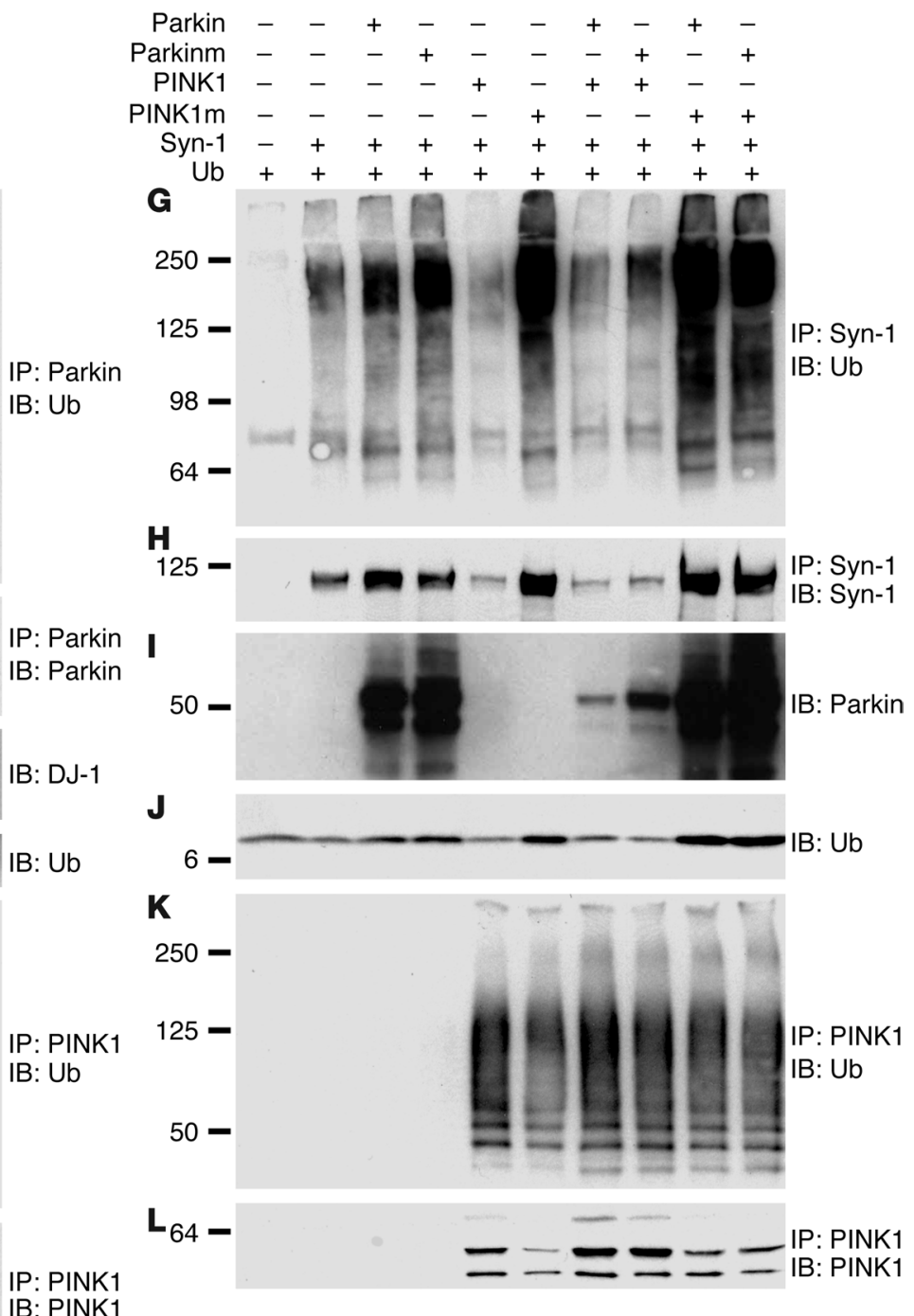

IB: PINK1

\section{Figure 4}

PINK1 regulates ubiquitination of Parkin and Synphilin-1. Parkin (left) or Synphilin-1 (right) was cotransfected into SH-SY5Y cells with PINK1WT, a PD-pathogenic PINK1G309D mutant (PINK1m), DJ-1WT, or a PD-pathogenic DJ-1L166P mutant (DJ-1m) in the presence of ubiquitin (Ub) in various combinations. In a Synphilin-1 degradation experiment, a PD-pathogenic ParkinR42P mutant (Parkinm) was included. Parkin (A and B) or Synphilin-1 ( $\mathbf{G}$ and $\mathbf{H}$ ) were immunoprecipitated from equal amounts of cell lysates, followed by detection of ubiquitin (A and $\mathbf{G})$, Parkin (B), or Synphilin-1 (H). Expression levels of DJ-1WT (C), DJ-1L166P (C), and ubiquitin (D and J) were shown by direct immunoblotting. Ubiquitination and steady-state levels of PINK1 variants were observed by immunoprecipitation of PINK1, followed by immunoblotting of either ubiquitin ( $E$ and $\mathbf{K}$ ) or PINK1 (F and L).

fected cells and an in vitro ubiquitination assay using previously defined $(10,11,26)$ Parkin substrates, Synphilin-1, and Parkin itself. Our results suggest that Parkin likely has very limited activity in the absence of PINK1. However, Parkin E3 ligase activity is remarkably potentiated by expression of PINK1. Thus, PINK1 plays an essential role in maintaining high E3 ligase activity of Parkin. These findings also provide an explanation for inconsistent reports of Parkin E3 ligase activity in previous studies (12). Another importance of this study is to identify the PPD complex as what we believe is a novel E3 ligase. Three classes of E3 ligase have been previously described, including HECT domain ligases, RING finger ligases, and U-box domain ligases (30). RING finger
E3 ligases can be either a single protein, such as CBL (31), or a multiple protein complex, such as the skip/cullin/F-box/Rbx $1 / 2$ (SCF) complex (32). In the PPD complex, Parkin is a RING finger protein binding to E2 and substrate, while PINK1 and DJ-1 act as regulatory components. PINK1, a serine/threonine kinase, makes the PPD complex, to our knowledge, the first E3 ligase complex with a kinase subunit. The role of serine/threonine kinase activity of PINK1 in this complex remains unknown. One possibility is that PINK1 regulates protein ubiquitination by phosphorylating either the complex components or substrates. DJ-1 facilitates but is not required for E3 ligase activity of the complex. One potential role of DJ-1 in this complex is to stabilize PINK1 (27). 


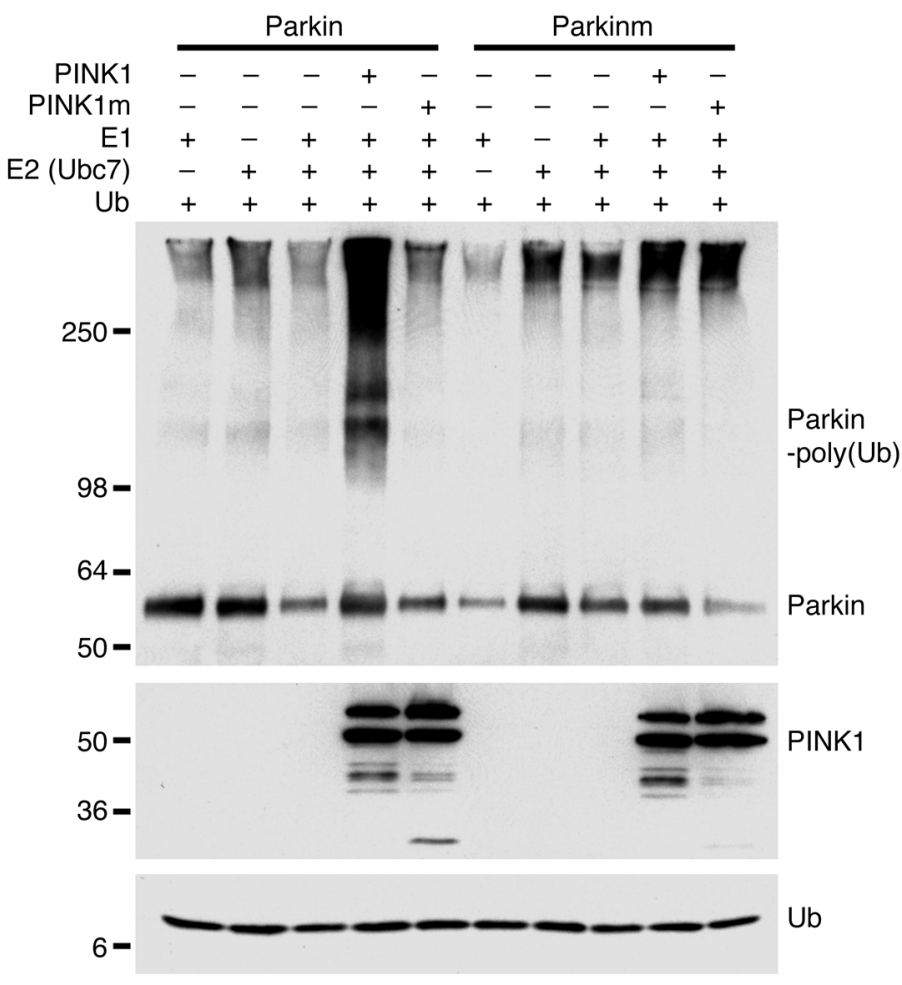

\section{Figure 5}

PINK1 promotes Parkin auto-ubiquitination in vitro. Affinity-purified ParkinWT, the PD-associated ParkinR42P mutant, PINK1WT, and PD-associated PINK1G309D mutant proteins in various combinations were assayed for in vitro ubiquitination in the presence of recombinant E1, E2 (Ubc7), and HA-tagged ubiquitin. Proteins were separated on SDS-PAGE and immunoblotted with an antiParkin antibody to detect Parkin monomers (Parkin) and ubiquitinated Parkin [Parkin-poly(Ub)], an anti-VSVG antibody to detect PINK1, or an anti-HA antibody to detect ubiquitin. Ubiquitinated Parkin appeared as a smear in the top panel.

Parkin poly(Ub)

arkin

The UPS plays critical roles in PD pathogenesis $(5,33,34)$. Our finding of PD-pathogenic mutants of PINK1 and Parkin to impair the PPD E3 ligase activity supports a role of the PPD E3 ligase in PD pathogenesis. Consistent with this notion, we have previous identified digenic mutations of PINK1 and DJ-1 that are associated with early onset familial PD cases (27). Since the identification of Parkin as an E3 ligase, one uncertainty is the identity of its substrates that are also the substrates of the PPD complex. Parkin ubiquitinates a number of proteins in vitro. Nevertheless, few of these in vitro substrates accumulate in Parkin-deficient cells (20). In this study, we have also found that levels of Parkin and Synphilin-1 are not altered in either PINK1- or DJ-1-deficient cells. These results suggest that Parkin substrates can be degraded by alternative mechanisms under physiological conditions. However, overexpression of Parkin substrates or heat shock treatment resulted in Parkin substrate accumulation in PINK1-deficient cells. One explanation is that the PPD complex functions as a quality control system to degrade unfolded and misfolded Parkin substrates induced by oxidative stress in neurons. Pathogenic mutations that reduce PPD activity likely disrupt the UPS, leading to increased susceptibility to oxidative stress and accumulation of un-/misfolded proteins in cells, therefore eventual neuronal death and Lewy body formation.

\section{Figure 6}

Ubiquitination of endogenous Parkin in mouse brains with PINK1 and DJ-1 ablation. Top: Brain slices from WT and PINK1-deficient (KO) mice were immunoprecipitated with either a monoclonal anti-Parkin antibody or a control mouse IgG, followed by immunoblotting with an anti-ubiquitin antibody. Cells overexpressing exogenous Parkin were used as a positive control. The experiments were done with or without heat shock treatment (HS). Bottom: Immunoprecipitated Parkin protein was detected by anti-Parkin polyclonal antibody. The 55-kDa band shown in control precipitations were IgG heavy chain. Note that Parkin ubiquitination was remarkably reduced in PINK1-null brain slices.

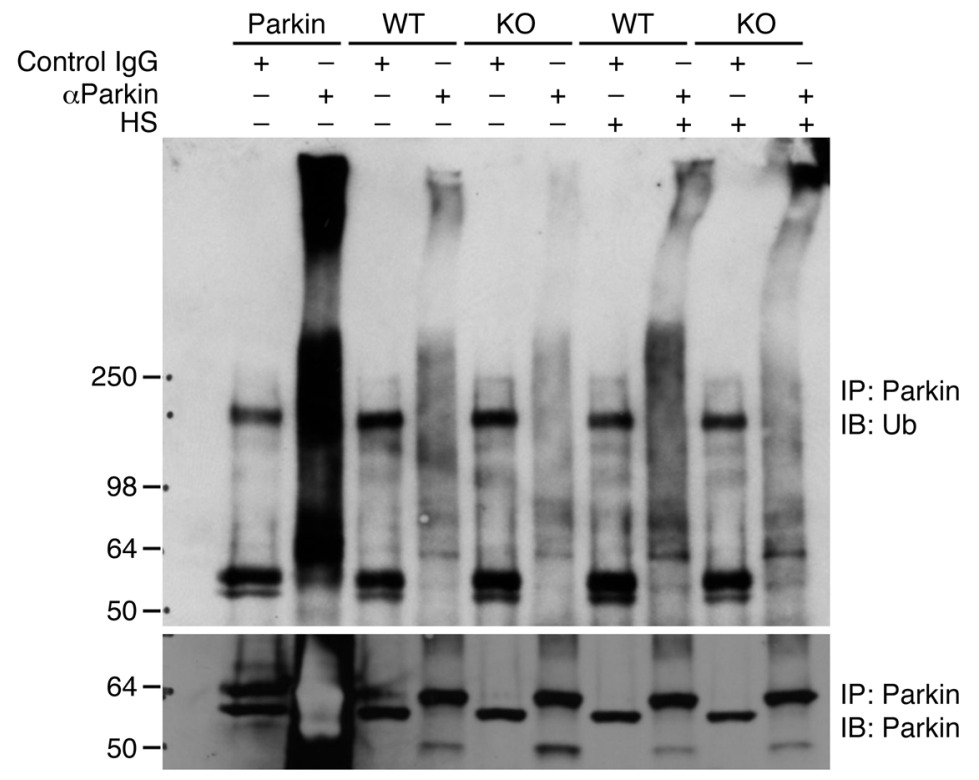



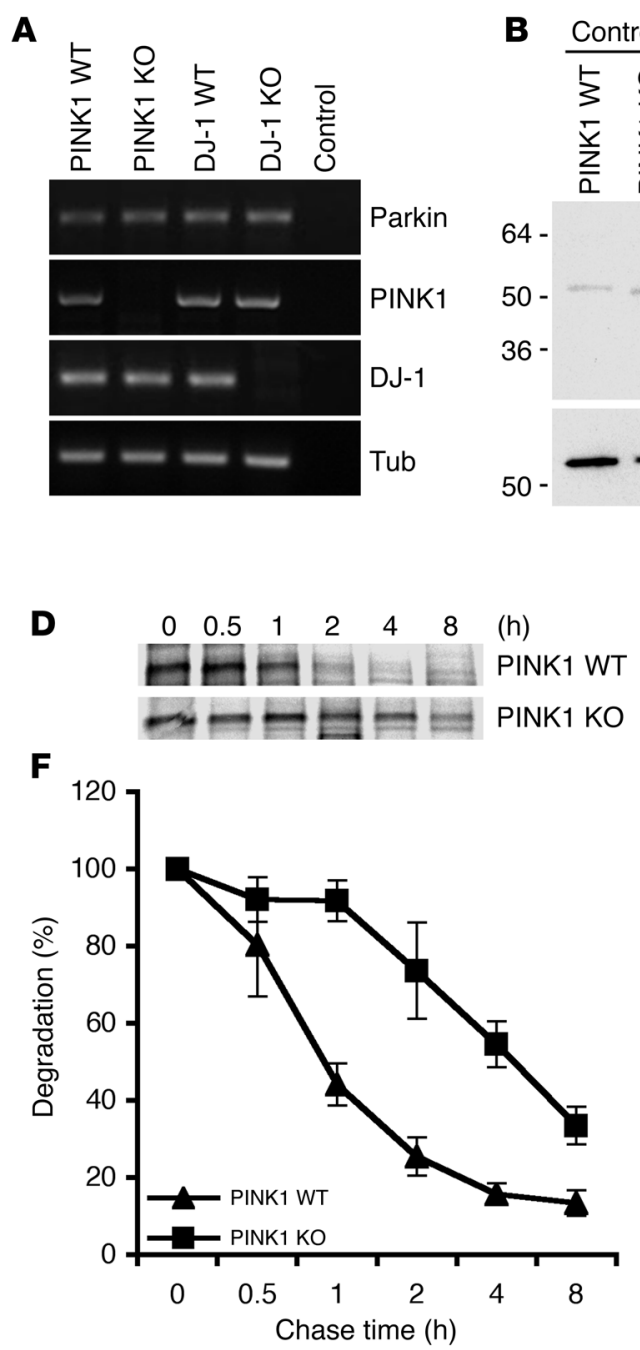
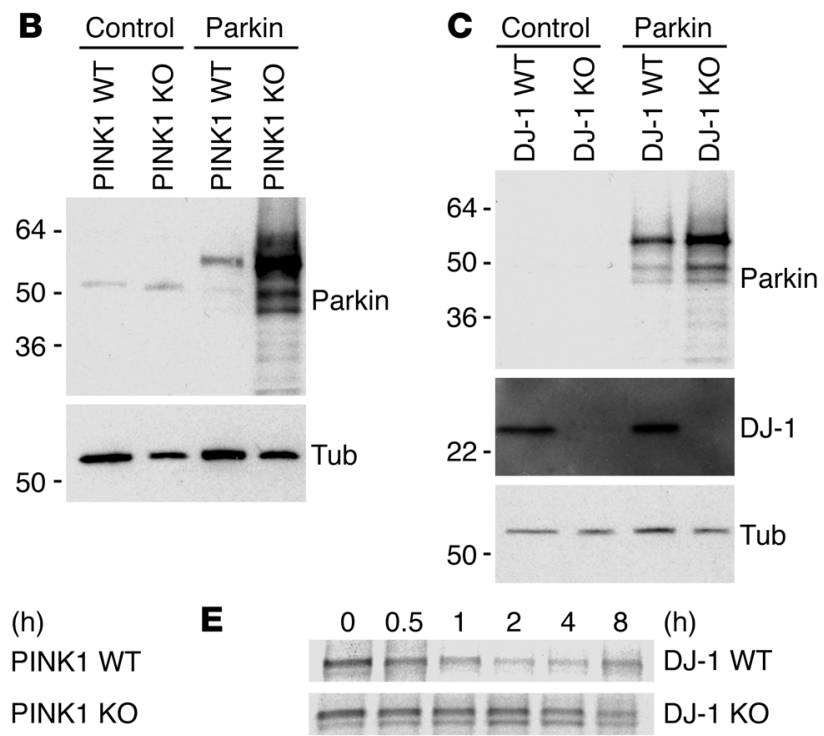

G

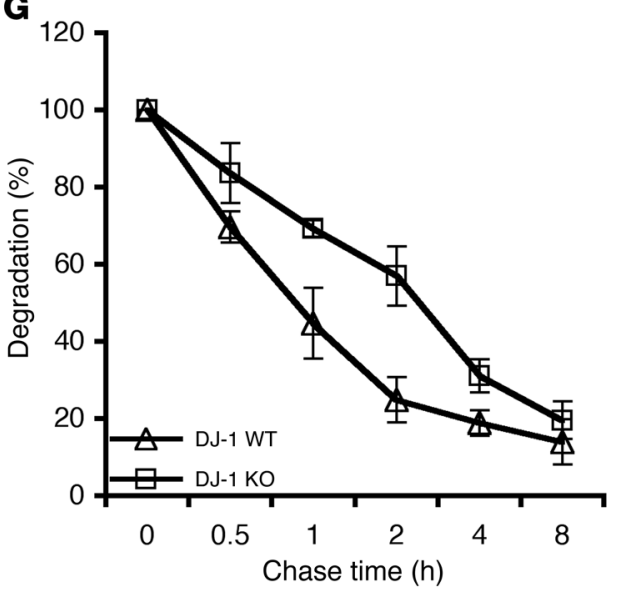

Figure 7

Genetic ablation of mouse Pink1 or Dj-1 results in increased stability of aberrantly expressed Parkin. (A) Expression of Parkin, PINK1, and DJ-1 in PINK1 - or DJ-1-deficient mouse fibroblasts. RT-PCR detection of Parkin, PINK1, and DJ-1 in PINK1 WT, PINK1 KO, DJ-1 WT, and DJ-1 KO cells. Control, no cDNA template added. (B and C) Increased accumulation of aberrantly expressed Parkin in PINK1 KO and DJ-1 KO cells. Cells transfected with control plasmid or plasmid encoding Parkin showed increased Parkin detection in PINK1 KO cells (B) and DJ-1 KO cells (C). (C) Tubulin was used as a control. Lack of DJ-1 protein in DJ-1 KO cells was shown by immunoblotting. (D-G) Increased stability of Parkin in PINK1 KO and DJ-1 KO cells. PINK1 KO, PINK1 WT, DJ-1 KO, and DJ-1 WT cells were transfected with Parkin, followed pulse chase analysis of Parkin stability for the time frames indicated. Representative results of PINK1 (D) and DJ-1 (E) are shown. Quantitation was obtained from PINK1 KO cells generated from 2 independent PINK1 KO mice (F) and DJ-1 KO cells generated from multiple DJ-1 KO mice (G).

WT Parkin, but not PD-pathogenic mutant Parkin, has been previously shown to protect cells against dopamine-induced oxidative stress via a UPS-mediated mechanism (35). Parkin, PINK1, and DJ-1 are also individually implicated in oxidative stress protection $(2,18,19,21,36-39)$. Furthermore, Parkin mediates polyubiquitination, and subsequently degradation, of the highly aggregative ER transmembrane protein Pael-R $(12,40)$. In this study, the aberrantly expressed proteins and heat shock-induced misfolded Parkin accumulated in the absence of PINK1.

This study connects 3 PD-linked genes in a single functional pathway that further highlights a common mechanism of PD pathogenesis. Parkin has been shown to ubiquitinate Synphilin-1 and a rare $O$-glycosylated form of $\alpha$-synuclein $(26,41)$, suggesting that these genes functionally interact to regulate $\mathrm{PD}$ pathogenesis.
A recent study indicates a role for Parkin in modulating ubiquitination of LRRK2, a newly identified gene linked to autosomal dominant familial PD (42). Together, this study should facilitate further investigation of PD-pathogenic mechanisms and the design of novel PD treatment strategies.

\section{Methods}

Mice, cell lines, plasmids, and siRNAs. The DJ-1-deficient mouse line has been described previously (28). The Pink1-deficient mice were generated by replacing a 5.6-kb genomic region in the Pink1 locus, including exons 4-7 and the coding portion of exon 8, with FRT sequences flanked PGK-neo-polyA selection cassette. Both the $3.5-\mathrm{kb}$ and $4.8-\mathrm{kb}$ homologous arms were amplified by PCR using genomic DNA isolated from E14Tg2A.4 embryonic stem cells (BayGenomics) as a template. E14Tg2A.4 embryonic stem cells were electro- 


$\begin{array}{rcccccccccc} & 1 & 2 & 3 & 4 & 5 & 6 & 7 & 8 & 9 & 10 \\ \text { Parkin } & - & + & + & + & + & + & + & + & + & + \\ \text { PINK1 } & - & - & - & - & - & - & - & + & + & + \\ \text { Ub } & - & - & - & - & + & + & + & + & + & + \\ \text { HS } & - & - & + & + & - & + & + & - & + & + \\ \text { RT } & 0 & 0 & 0 & 4 & 0 & 0 & 4 & 0 & 0 & 4\end{array}$

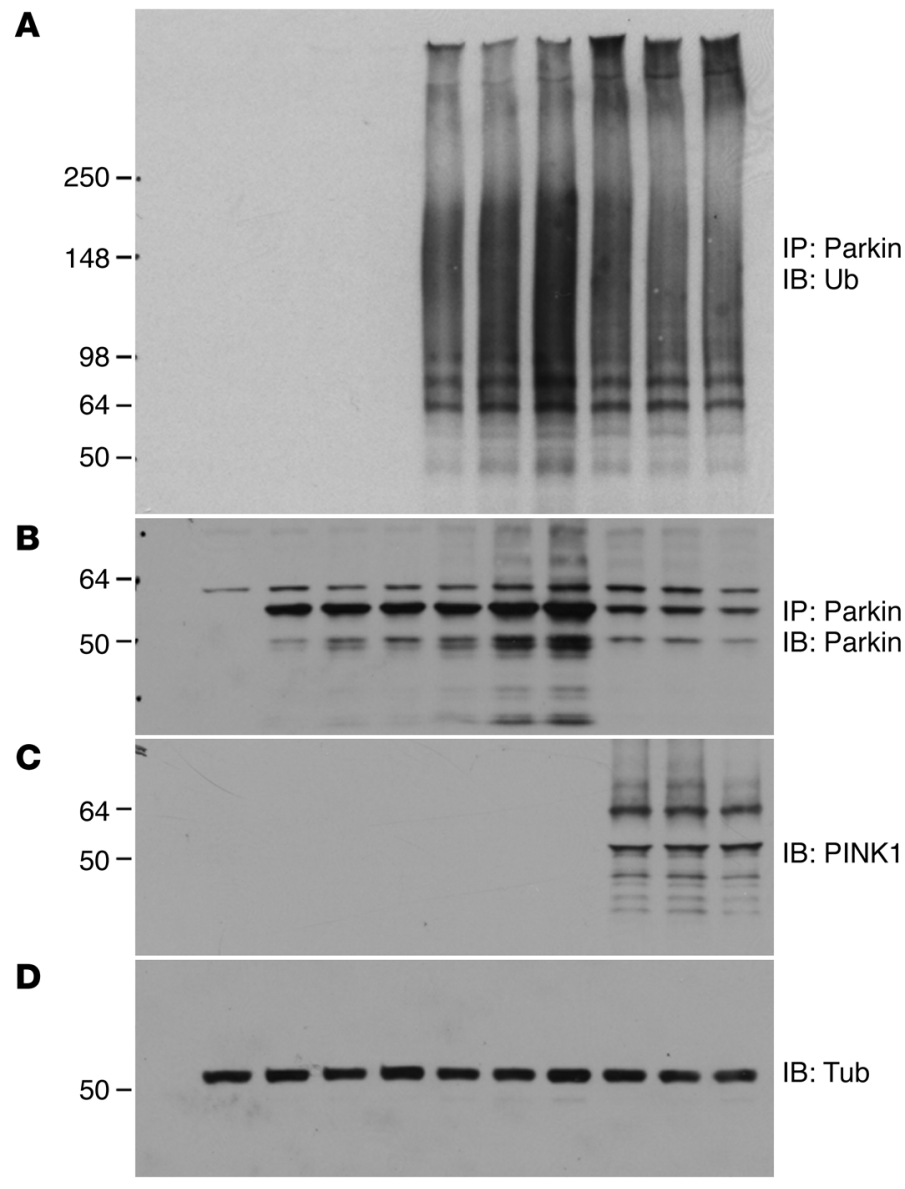

Figure 8

PINK1 promotes degradation of Parkin that has accumulated as a result of heat shock treatment. Cells expressing Parkin alone (lanes 2-4); Parkin and ubiquitin (lanes 5-7); and Parkin, ubiquitin, and PINK1 (lanes 8-10) without heat shock treatment (lanes 1, 2, 5, 8), with heat shock treatment (lanes 3 , 6,9 ), or with heat shock treatment followed by a 4-h recovery time (RT) at $37^{\circ} \mathrm{C}$ (lanes $4,7,10$ ). The cells were lysed and immunoprecipitated with an anti-Parkin antibody, followed by immunoblotting with either an anti-ubiquitin antibody (A) or an anti-Parkin antibody (B). Expression of PINK1 (C) and tubulin (D) were shown with immunoblotting. Note that heat shock treatment increased accumulation of Parkin protein (B; lanes 6-7). PINK1 promoted degradation of Parkin even with heat shock treatment (B; lanes 9-10).

porated $(800 \mathrm{~V}$ and $3 \mu \mathrm{F})$ with $30 \mu \mathrm{g}$ of linearized targeting construct. G418resistant clones were screened by Southern blotting for homologous recombination with a $5^{\prime}$ external probe. Positive cells were injected into C57BL6/J blastocysts to generate chimeras, which were then mated with C57BL6/J WT mice to generate heterozygotes. Heterozygous mice on a $129 \times \mathrm{C} 57 \mathrm{BL} / 6$ mixed background were bred to generate Pink1-null mice and their WT littermate controls for experiments. Mice were genotyped by multiplex PCR on genomic DNA extracted from tail snips: the first primer pair amplified part of intron 6 of Pink1 (present in all mice), the second primer pair amplified part of neo ${ }^{\mathrm{r}}$ (absent in WT mice), and the third primer pair amplified intron 6 of PINK1 (absent in homozygous mutants). The absence of PINK1 expression was verified by RT-PCR and in situ hybridization (data not shown). All animal procedures were approved by the Institutional Animal Care and Usage Committees (IACUC) of the University of Chicago and the Burnham Institute for Medical Research.

Mouse embryonic fibroblasts were generated from PINK1- and DJ-1deficient mouse embryos and their WT control littermates. Cells were immortalized with a retrovirus expressing SV40 large T antigen. SH-SY5Y neuroblastoma cells and HEK293 cells were purchased from ATCC. cDNAs encoding myc-tagged, flag-tagged, or VSVGtagged Parkin variants (WT, $\triangle \mathrm{E} 4, \mathrm{R} 42 \mathrm{P}$, and T240W), PINK1 variants (WT, G309D, T313M, L339P) and DJ-1 variants (WT and L166P) were PCR generated and subcloned into pcDNA3.1(-) (Invitrogen). A SMARTpool of siRNAs for human Parkin was purchased from Dharmacon. Plasmids encoding HA-ubiquitin and myc-ubiquitin were previously described (43). GFP-HA-tagged Synphilin-1 was a gift of Stuart Lipton at the Burnham Institute for Medical Research. All constructs were confirmed by sequencing.

Antibodies and immunoassays. A monoclonal antibody against DJ-1 (E2.19) was purchased from Signet Laboratories. Polyclonal anti-Parkin antibody was from Cell Signaling Technology. Anti-Parkin monoclonal antibody, anti-myc-tagged monoclonal antibody (9E10.2), agarose conjugated with a polyclonal anti-myc antibody, monoclonal antibodies against VSVG (P4D5) and FLAG (M2) tags and their conjugated agarose were from Sigma-Aldrich. A polyclonal anti-PINK1 antibody was from Imgenex. Immunoblotting and immunoprecipitation were performed as previously described (44). Treatment with MG132 (5-10 $\mu \mathrm{M})$ and leupeptin $(50 \mu \mathrm{M})$ was performed at $37^{\circ} \mathrm{C}$ overnight. Heat shock was performed at $42^{\circ} \mathrm{C}$ for $2 \mathrm{~h}$ followed by recovery at $37^{\circ} \mathrm{C}$.

Generation of Parkin, PINK1, and DJ-1 fusion proteins. cDNAs encoding tagged Parkin (Parkin-flag-myc) and PINK1 (PINK1-flagVSVG) were cloned into $\mathrm{pFastBac1}$ vectors and transformed into DH10Bac competent cells. Individual recombinant bacmid DNAs were then transfected into Sf9 insect cells using Superfect transfection reagent (Qiagen). Recombinant baculovirus was then transferred and amplified in Sf9 cells. Cell pellets were collected after $48 \mathrm{~h}$ followed by affinity purification using anti-flag antibody (M2) conjugated to agarose and eluted with flag peptides according to the manufacturer's instructions (Sigma-Aldrich).

To generate DJ-1 fusion proteins, cDNA encoding DJ-1-VSVG was subcloned into pGEX4T2. Protein expression and purification were done according to the manufacturer's instructions (GE).

Parkin, PINK1, and DJ-1 complex assembly in vitro. Affinity-purified Parkin-myc-flag $(0.1 \mu \mathrm{g})$, PINK1-VSVG-flag $(0.1 \mu \mathrm{g})$, and GST-DJ-1VSVG $(0.2 \mu \mathrm{g})$ fusion proteins were added to $400 \mu \mathrm{l}$ binding buffer (10 mM HEPES, pH 7.5, $142.4 \mathrm{mM} \mathrm{KCl,} 5 \mathrm{mM} \mathrm{MgCl}_{2}, 1 \mathrm{mM}$ EGTA, $1 \% \mathrm{NP}-40,5 \% \mathrm{BSA}$, and $10 \%$ glycerol) in various combinations. Antimyc beads $(15 \mu \mathrm{l})$ and $15 \mu \mathrm{l} \mathrm{GST}$ beads were included in the binding assay to precipitate Parkin and DJ-1, respectively. As an internal control, $0.2 \mu \mathrm{g}$ of his-KChIP1 fusion protein was added to each assay. Mixtures were rotated at room temperature for $3 \mathrm{~h}$. Beads were washed 5 times with $1 \mathrm{ml}$ of BSA-free binding buffer. Proteins binding to beads were separated on $4 \%-20 \%$ Tris-Glycine gels and detected by immunoblotting with corresponding antibodies.

Mitochondrial and cytosolic fractionation. Isolation of mitochondria and cytosolic fraction from cells expressing Parkin, PINK1, and DJ-1 was done as previously described (45).

Metabolic labeling and pulse-chase analysis. Cells were metabolically labeled with ${ }^{35}$ S-protein labeling mix for $15-25 \mathrm{~min}$, followed by a chase with $\mathrm{DMEM} / 10 \% \mathrm{FBS} / 3 \mathrm{mM}$ unlabeled methionine for various time periods. For MG132 treatment, cells were pretreated $(5 \mu \mathrm{M})$ for $3 \mathrm{~h}$ followed by a 
A

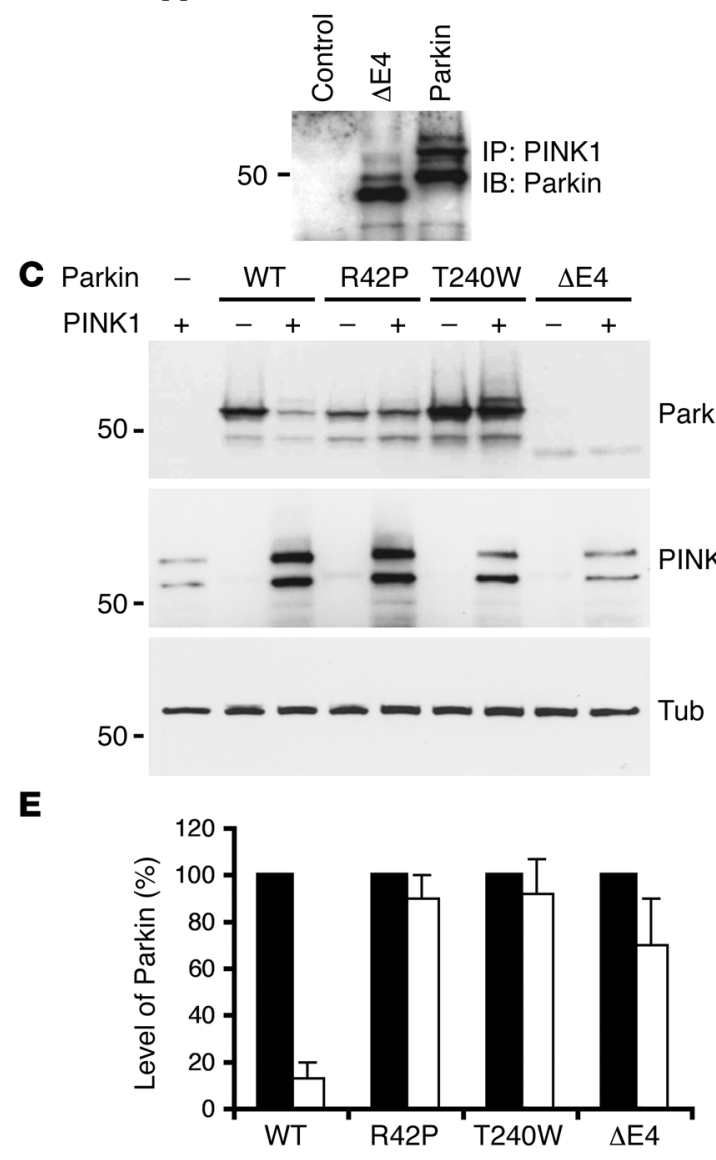

B

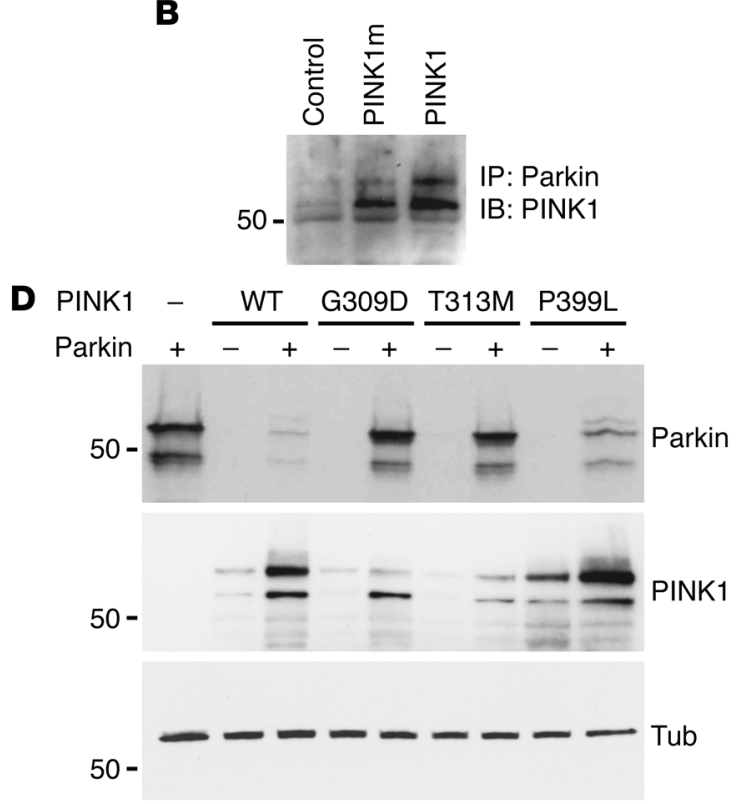

$\mathbf{F}$

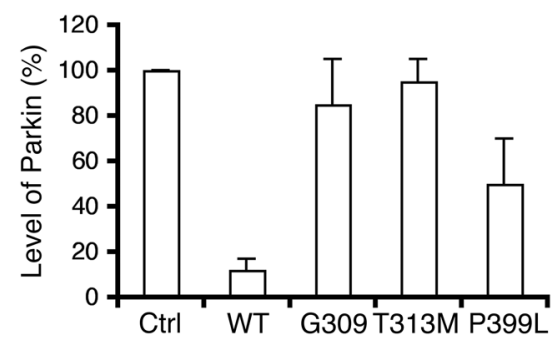

Figure 9

PD-pathogenic Parkin or PINK1 mutants impair Parkin degradation. (A) Interaction of PINK1WT with PD-pathogenic Parkin $\Delta$ E4. Cells expressing PINK1WT alone (Control), PINK1WT and Parkin $\Delta \mathrm{E} 4(\Delta \mathrm{E} 4)$, or PINK1WT and ParkinWT (Parkin) were immunoprecipitated with an anti-flag antibody (to precipitate PINK1), followed by immunoblotting with an anti-myc antibody (to detect Parkin variants). (B) Interaction of ParkinWT with PINK1G309D. Cells expressing ParkinWT alone (control), ParkinWT and PINK1G309D (G309D), or ParkinWT and PINK1WT (PINK1) were immunoprecipitated with anti-myc antibody (to precipitate Parkin), followed by immunoblotting with an anti-flag antibody (to detect PINK1 variants). (C) PINK1 promoted degradation of PD-pathogenic Parkin mutants. ParkinWT (WT) and PD-pathogenic ParkinR42P, -T240W, and - $\Delta$ E4 were cotransfected with or without PINK1. Steady-state levels of Parkin, PINK1, and tubulin were analyzed by immunoblotting. PINK1 promoted significant degradation of ParkinWT but not ParkinR42P, -T240W, or - $\triangle E 4$. (D) PD-pathogenic PINK1 mutants were impaired in promoting Parkin degradation. PINK1WT (WT) and PD-pathogenic PINK1G309D, -T313M, and -P399L were cotransfected with or without Parkin. Steady-state levels of Parkin, PINK1, and tubulin were detected by immunoblotting. PD-pathogenic mutants showed little or reduced ability to promote Parkin degradation. (E and F) Quantitation of Parkin degradation affected by pathogenic Parkin (E) or PINK1 (F) mutations. The data were from 3 independent experiments. Relative Parkin levels were normalized to either the level of Parkin variants without PINK1 expression (E, black bars) or the level of ParkinWT without PINK1 expression (Ctrl; F) in the same experiment.

chase in medium containing $5 \mu \mathrm{M} \mathrm{MG} 132$. Cells were lysed in $1 \mathrm{ml}$ RIPA buffer. Cell lysates were immunoprecipitated with respective antibodies. The results were quantified using a phosphorimager.

Protein ubiquitination in transfected cells. Ubiquitination was analyzed as previously described (46). Briefly, cells transfected with various plasmid combinations were lysed in $2 \%$ SDS buffer ( $2 \%$ SDS, $150 \mathrm{mM} \mathrm{NaCl}$, $10 \mathrm{mM}$ Tris- $\mathrm{HCl}, \mathrm{pH} 8.0,2 \mathrm{mM}$ sodium orthovanadate, $50 \mathrm{mM}$ sodium fluoride, $1 \times$ protease inhibitors) and boiled for $10 \mathrm{~min}$ followed by sonication. Lysates were diluted 1:10 in dilution buffer $(10 \mathrm{mM}$ Tris- $\mathrm{HCl}$, pH 8.0, $150 \mathrm{mM} \mathrm{NaCl}, 2 \mathrm{mM}$ EDTA, $1 \%$ Triton X-100), incubated at $4{ }^{\circ} \mathrm{C}$ for $1 \mathrm{~h}$ with rotation, and centrifuged at $20,800 \mathrm{~g}$ for $30 \mathrm{~min}$. Equal amounts $(1-1.5 \mathrm{mg})$ of protein were used for immunoprecipitation. Immunoprecipitated proteins were washed with washing buffer $(10 \mathrm{mM}$ Tris-HCl, $\mathrm{pH}$ 8.0, $1 \mathrm{M} \mathrm{NaCl}, 1 \mathrm{mM}$ EDTA, 1\% NP-40), boiled in SDS sample buffer, and separated on SDS-PAGE. Ubiquitin and precipitated proteins were immunodetected with antibodies.

In vitro ubiquitination assay. Affinity-purified Parkin-flag-myc $(0.2 \mu \mathrm{g})$, PINK1-flag-VSVG $(0.2 \mu \mathrm{g}), 10 \mathrm{ng}$ recombinant mouse E1 (10 ng) and GST-ubc7 (500 ng) were added to $40 \mu \mathrm{l}$ ubiquitination reactions $(50 \mathrm{mM}$ Tris- $\mathrm{HCl}$, pH 7.4, 5 mM MgCl $2,10 \mathrm{nM}$ okadaic acid, $0.6 \mathrm{mM}$ DTT, $2 \mathrm{mM}$ $\mathrm{NaF}, 2 \mathrm{mM}$ ATP, $0.03 \mathrm{U} / \mu \mathrm{l}$ creatine phosphokinase, $30 \mathrm{mM}$ creatine phosphate) and $100 \mathrm{ng} / \mu \mathrm{l}$ bovine ubiquitin (Sigma-Aldrich) in various combinations. Reactions proceeded for $1 \mathrm{~h}$ at $37^{\circ} \mathrm{C}$ and were stopped by addition of SDS-PAGE sample buffer. Proteins were separated on a $4 \%-20 \%$ SDS-PAGE gel and immunoblotted with anti-Parkin or anti-VSVG antibodies.

Mouse brain slice culture. Mesencephalon brain slice culture was prepared from brains of P4 or P5 PINK1-null mice and their control littermates as previously described with minor modifications (47). Briefly, 300- $\mu \mathrm{m}$-thick coronal mes- 
encephalonal sections were prepared using a Mcllwain tissue chopper (Mickle Laboratory Engineering Co.) and separated in ice-cold slice culture medium consisting of $50 \%$ minimum essential medium, 25\% HBSS, 25\% heat-inactivated horse serum supplemented with $36 \mathrm{mM}$ glucose, $25 \mathrm{mM}$ HEPES, $4 \mathrm{mM}$ $\mathrm{NaHCO}_{3}, 1 \%$ fungizone, $100 \mathrm{U} / \mathrm{ml}$ penicillin, and $100 \mu \mathrm{g} / \mathrm{ml}$ streptomycin, $\mathrm{pH}$ 7.2. Slices were placed on Millicell culture inserts and cultured in 6-well culture plates. Organotypic cultures were maintained in an incubator gasified with a $5 \%$ $\mathrm{CO}_{2} / 95 \% \mathrm{O}_{2}$ atmosphere at $37^{\circ} \mathrm{C}$, and the medium was changed 3 times/week. Seven days after the cultures were established, half of the slices were treated with heat shock in a $42^{\circ} \mathrm{C}$ incubator for $2 \mathrm{~h}$ followed by recovery at $37^{\circ} \mathrm{C}$ for an additional $4 \mathrm{~h}$. Endogenous Parkin was immunoprecipitated with a monoclonal anti-Parkin antibody (Sigma-Aldrich). Endogenous ubiquitin was detected with a rabbit monoclonal anti-ubiquitin antibody (Epitomics).

Statistics. Protein expression levels were quantified using ImageJ. All quantitative data were analyzed with the 2-tailed Student's $t$ test using InStat version 3 (GraphPad). All error bars indicate SEM.

\section{Acknowledgments}

This work was supported by NIH grants RO1 DC006497, RO1 NS057289, and PO1 ES016738 (to Z. Zhang); California Institute for Regenerative Medicine grants RS1-00331-1 and RL1-00682-1 (to Z. Zhang); Chinese Natural Science Foundation grants (to Z. Zhang); the American Parkinson Disease Association (to Z. Zhang and Y.S. Choo); and the Michael J. Fox Foundation for Parkinson's Research (to X. Zhuang and Z. Zhang).

Received for publication October 1, 2008, and accepted in revised form January 7, 2009.

Address correspondence to: Zhuohua Zhang, Burnham Institute for Medical Research, 10901 N. Torrey Pines Road, La Jolla, California 92037, USA. Phone: (858) 795-5286; Fax: (858) 646-3198; E-mail: benzz@burnham.org.
1. Dawson, T.M., and Dawson, V.L. 2003. Molecularpathways of neurodegeneration in Parkinson's disease. Science. 302:819-822.

2. Valente, E.M., et al. 2004. Hereditary early-onset Parkinson's disease caused by mutations in PINK1. Science. 304:1158-1160.

3. Paisan-Ruiz, C., et al. 2004. Cloning of the gene containing mutations that cause PARK8-linked Parkinson's disease. Neuron. 44:595-600.

4. Zimprich, A., et al. 2004. Mutations in LRRK2 cause autosomal-dominant parkinsonism with pleomorphic pathology. Neuron. 44:601-607.

5. Cookson, M.R. 2005. The biochemistry of Parkinson's disease. Annu. Rev. Biochem. 74:29-52.

6. Ramirez, A., et al. 2006. Hereditary parkinsonism with dementia is caused by mutations in ATP13A2, encoding a lysosomal type 5 P-type ATPase. Nat. Genet. 38:1184-1191.

7. Spillantini, M.G., et al. 1997. Alpha-synuclein in Lewy bodies. Nature. 388:839-840.

8. Conway, K.A., et al. 2000. Acceleration of oligomerization, not fibrillization, is a shared property of both alpha-synuclein mutations linked to earlyonset Parkinson's disease: implications for pathogenesis and therapy. Proc. Natl. Acad. Sci. U. S. A. 97:571-576.

9. Conway, K.A., Harper, J.D., and Lansbury, P.T. 1998. Accelerated in vitro fibril formation by a mutant alpha-synuclein linked to early-onset Parkinson disease. Nat. Med. 4:1318-1320.

10. Shimura, H., et al. 2000. Familial Parkinson disease gene product, parkin, is a ubiquitin-protein ligase. Nat. Genet. 25:302-305.

11. Zhang, Y., et al. 2000. Parkin functions as an E2dependent ubiquitin- protein ligase and promotes the degradation of the synaptic vesicle-associated protein, CDCrel-1. Proc. Natl. Acad. Sci. U. S. A. 97:13354-13359.

12. Imai, Y., Soda, M., and Takahashi, R. 2000. Parkin suppresses unfolded protein stress-induced cell death through its E3 ubiquitin-protein ligase activity. J. Biol. Chem. 275:35661-35664.

13. Valente, E.M., et al. 2004. PINK1 mutations are associated with sporadic early-onset parkinsonism. Ann. Neurol. 56:336-341.

14. Plun-Favreau, H., et al. 2007. The mitochondrial protease HtrA2 is regulated by Parkinson's diseaseassociated kinase PINK1. Nat. Cell Biol. 9:1243-1252.

15. Pridgeon, J.W., Olzmann, J.A., Chin, L.S., and Li, L. 2007. PINK1 protects against oxidative stress by phosphorylating mitochondrial chaperone TRAP1. PLoS Biol. 5:e172.

16. Bonifati, V., et al. 2003. Mutations in the DJ-1 gene associated with autosomal recessive early-onset parkinsonism. Science. 299:256-259.
17. Taira, T., et al. 2004. DJ-1 has a role in antioxidative stress to prevent cell death. EMBO Rep. 5:213-218.

18. Menzies, F.M., Yenisetti, S.C., and Min, K.T. 2005. Roles of Drosophila DJ-1 in survival of dopaminergic neurons and oxidative stress. Curr. Biol. 15:1578-1582.

19. Meulener, M., et al. 2005. Drosophila DJ-1 mutants are selectively sensitive to environmental toxins associated with Parkinson's disease. Curr. Biol. 15:1572-1577.

20. Goldberg, M.S., et al. 2003. Parkin-deficient mice exhibit nigrostriatal deficits but not loss of dopaminergic neurons. J. Biol. Chem. 278:43628-43635.

21. Palacino, J.J., et al. 2004. Mitochondrial dysfunction and oxidative damage in parkin-deficient mice. J. Biol. Chem. 279:18614-18622.

22. Clark, I.E., et al. 2006. Drosophila pink1 is required for mitochondrial function and interacts genetically with parkin. Nature. 441:1162-1166.

23. Park, J., et al. 2006. Mitochondrial dysfunction in Drosophila PINK1 mutants is complemented by parkin. Nature. 441:1157-1161.

24. Wang, D., et al. 2006. Antioxidants protect PINK1dependent dopaminergic neurons in Drosophila. Proc. Natl. Acad. Sci. U. S. A. 103:13520-13525.

25. Yang, Y., et al. 2006. Mitochondrial pathology and muscle and dopaminergic neuron degeneration caused by inactivation of Drosophila Pink1 is rescued by Parkin. Proc. Natl. Acad. Sci. U. S. A. 103:10793-10798.

26. Chung, K.K., et al. 2001. Parkin ubiquitinates the alpha-synuclein-interacting protein, synphilin-1: implications for Lewy-body formation in Parkinson disease. Nat. Med. 7:1144-1150.

27. Tang, B., et al. 2006. Association of PINK1 and DJ-1 confers digenic inheritance of early-onset Parkinson's disease. Hum. Mol. Genet. 15:1816-1825.

28. Chen, L., et al. 2005. Age-dependent motor deficits and dopaminergic dysfunction in DJ-1 null mice. J. Biol. Chem. 280:21418-21426.

29. Beilina, A., et al. 2005. Mutations in PTEN-induced putative kinase 1 associated with recessive parkinsonism have differential effects on protein stability. Proc. Natl. Acad. Sci. U. S. A. 102:5703-5708.

30. Glickman, M.H., and Ciechanover, A. 2002. The ubiquitin-proteasome proteolytic pathway: destruction for the sake of construction. Physiol. Rev. 82:373-428.

31. Joazeiro, C.A., et al. 1999. The tyrosine kinase negative regulator c-Cbl as a RING-type, E2-dependent ubiquitin-protein ligase. Science. 286:309-312.

32. Petroski, M.D., and Deshaies, R.J. 2005. Function and regulation of cullin-RING ubiquitin ligases. Nat. Rev. Mol. Cell Biol. 6:9-20.

33. Hattori, N., and Mizuno, Y. 2004. Pathogenetic mechanisms of parkin in Parkinson's disease. Lancet. 364:722-724

34. Moore, D.J., West, A.B., Dawson, V.L., and Dawson, T.M. 2005. Molecular pathophysiology of Parkinson's disease. Annu. Rev. Neurosci. 28:57-87.

35. Jiang, H., Ren, Y., Zhao, J., and Feng, J. 2004. Parkin protects human dopaminergic neuroblastoma cells against dopamine-induced apoptosis. Hum. Mol. Genet. 13:1745-1754.

36. Greene, J.C., et al. 2003. Mitochondrial pathology and apoptotic muscle degeneration in Drosophila parkin mutants. Proc. Natl. Acad. Sci. U. S. A. 100:4078-4083.

37. Pesah, Y., et al. 2004. Drosophila parkin mutants have decreased mass and cell size and increased sensitivity to oxygen radical stress. Development. 131:2183-2194.

38. Kim, R.H., et al. 2005. Hypersensitivity of DJ-1deficient mice to 1-methyl-4-phenyl-1,2,3,6-tetrahydropyrindine (MPTP) and oxidative stress. Proc. Natl. Acad. Sci. U. S. A. 102:5215-5220.

39. Moore, D.J., et al. 2005. Association of DJ-1 and parkin mediated by pathogenic DJ-1 mutations and oxidative stress. Hum. Mol. Genet. 14:71-84.

40. Imai, Y., et al. 2002. CHIP is associated with Parkin, a gene responsible for familial Parkinson's disease, and enhances its ubiquitin ligase activity. Mol. Cell. 10:55-67.

41. Shimura, H., et al. 2001. Ubiquitination of a new form of alpha-synuclein by parkin from human brain: implications for Parkinson's disease. Science. 293:263-269.

42. Smith, W.W., et al. 2005. Leucine-rich repeat kinase 2 (LRRK2) interacts with parkin, and mutant LRRK2 induces neuronal degeneration. Proc. Natl. Acad. Sci. U. S. A. 102:18676-18681.

43. Habelhah, H., et al. 2004. Ubiquitination and translocation of TRAF2 is required for activation of JNK but not of p38 or NF-kappaB. EMBO J. 23:322-332.

44. Zhang, Z., et al. 1998. Destabilization of betacatenin by mutations in presenilin- 1 potentiates neuronal apoptosis. Nature. 395:698-702.

45. Choo, Y.S., Johnson, G.V., MacDonald, M., Detloff, P.J., and Lesort, M. 2004. Mutant huntingtin directly increases susceptibility of mitochondria to the calcium-induced permeability transition and cytochrome c release. Hum. Mol. Genet. 13:1407-1420.

46. Didier, C., et al. 2003. RNF5, a RING finger protein that regulates cell motility by targeting paxillin ubiquitination and altered localization. Mol. Cell. Biol. 23:5331-5345.

47. Stoppini, L., Buchs, P.A., and Muller, D. 1991. A simple method for organotypic cultures of nervous tissue. J. Neurosci. Methods. 37:173-182. 